\title{
Neuropsychological profile linked to low dopamine: in Alzheimer's disease, major depression, and Parkinson's disease
}

\author{
Nicola Wolfe, Douglas I Katz, Martin L Albert, Abraham Almozlino, Raymon Durso, \\ Marcia C Smith, Ladislav Volicer
}

\begin{abstract}
A distinct pattern of neuropsychological deficits was associated with low homovanillic acid (HVA) in the cerebrospinal fluid of 21 patients with: Alzheimer's disease (9), Parkinson's disease (8) and major depressive disorders (4). Regardless of clinical diagnosis, patients with low HVA were slower on a test of efficiency of processing timed information, and showed greater benefit from semantic structure on a verbal fluency task than patients with high HVA. However, low HVA subjects were not significantly impaired on confrontation naming (Boston Naming Test). Across three diagnostic groups, patients with lower HVA also tended to have more extrapyramidal motor signs and were significantly more depressed. These results demonstrate a significant relationship between specific neurobehavioural deficits and dopaminergic activity which cuts across traditional diagnostic categories.
\end{abstract}

Based on a description of the role of dopamine in dementia by Bachman and Albert, ${ }^{1}$ we suspected that patients with dopamine deficiency might exhibit a characteristic neurobehavioural profile that cuts across the traditional diagnostic categories of Alzheimer's disease, Parkinson's disease, and major depressive disorders. Low dopamine has been associated with extrapyramidal motor signs, cognitive impairment and depression within each of these disorders. We suspected that low dopamine could be a common neurochemical pathology which accounts for similarities observed across these diagnostic groups. In this pilot study, we tested the hypothesis that regardless of diagnosis, subjects with low cerebrospinal fluid (CSF) homovanillic acid (HVA) would exhibit a syndrome consisting of the following: Parkinsonian motor signs, slowed cognition (bradyphrenia), and depression.

Patients and methods

We evaluated twenty one male inpatients with diagnoses of dementia of the Alzheimer type (9) (ADRDA-NINCDS criteria), ${ }^{2}$ Parkinson's disease (8) (clinical consensus by two neurologists on (a) the presence of at least three of the four cardinal features: resting temor, rigidity, bradykinesia, postural instability, and (b) absence of other known aetiologies of extrapyramidal symptoms), or major depressive disorder (4) (DSMIII). ${ }^{3}$ Exclusion criteria for all patients were: evidence of structural lesion on CT scan, Hachinski Ischaemia Score greater than 4 , or history of seizure. Neuropsychological testing included the following range of tests: the Mini-Mental State Test (MMS), a standard index of overall cognitive ability, and the Varied Interval Summation Task (VIST), a simplified version of the Paced Auditory Serial Addition Task ${ }^{4}$ designed to measure efficiency of processing speeded information. In this test, subjects hear a number (between 3 and 9) followed by a random series of the numbers 1 or 2 . The task is to add the series and report the sum after the tenth number. The interstimulus interval ranges from $5 \mathrm{~s}$ on the first trial to $0.7 \mathrm{~s}$ on the last. Thus successive trials increase the rate of presentation of stimuli, but the verbal response is not timed. The test score is the total number of successful trials. A pilot study of this instrument (Katz, unpublished data) showed it to be sensitive to efficiency of processing speeded information and to attentional deficits.

The Controlled Oral Word Association Test (FAS) of verbal fluency ${ }^{5}$ was used to assess benefit from semantic structure in word list generation. Benefit from structure was calculated as: number of correct responses in Semantic Categories (animals, fruits and vegetables, first names); number of correct responses in Phonemic Categories (starting with first letter F, A, and S successively)/ (Total correct responses). The Boston Naming Test (BNT) (30 item version) was included as an index of language function predicted to be uncorrelated with dopamine deficiency. Extrapyramidal motor signs were assessed with the Parkinson's Disease Motor Performance Scale (PDS), based on the scale by Duvoisin ${ }^{6}$; and affect was measured with the Hamilton Depression Rating Scale (HDRS). 
Figure Cognitive profiles of patients with low versus high CSF HVA.

Results in patients with lower $H V A(n=9)$, compared to high $H V A$ $(n=12)$. Statistically significant differences: ${ }^{\star} p<0.025,{ }^{\star \star} p<0.01$ (one-tailed).

*Benefit from semantic structure was computed as: (number correct using semantic categories minus number correct using phonemic categories)/ (total correct responses). Data in Table.

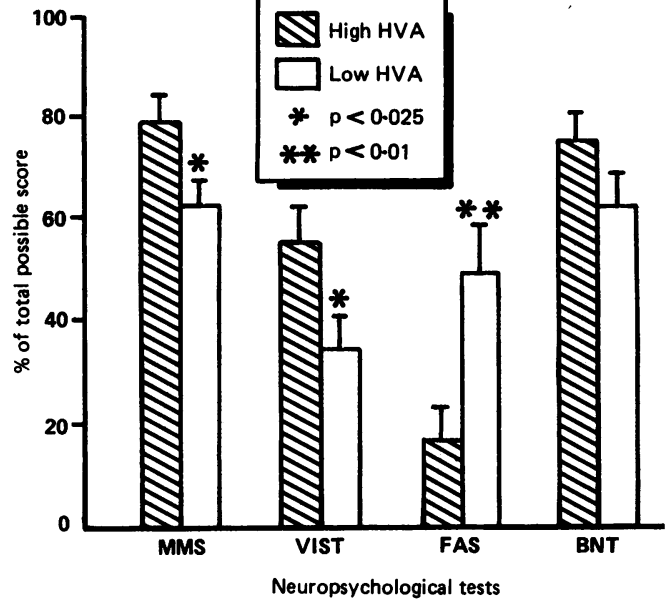

Lumbar puncture was performed using standard clinical procedure with the patient in the lateral recumbent position at 9 am after the patient had fasted and remained in bed from midnight of the previous day. CSF was quick frozen at the bedside and stored at $-70^{\circ} \mathrm{C}$ until the day of assay. HVA in the 7-9th cc was measured by high pressure liquid chromatography based on the method described by Matson et $\mathrm{al}^{7}$ using absolute coulometric detection with high selectivity and resolution. HVA levels obtained were comparable with other studies. $^{8}$

Most patients were not taking drugs, but $14 \%(3 / 21)$ had received major tranquilisers (minimum washout period 10 days) and $19 \%$ $(4 / 21)$ had previously received levodopa therapy (Sinemet for Parkinson's disease minimum washout period two days). The length of the washout period was not significantly correlated with CSF HVA in these patients, however, the number of these patients is too small to rule out the possibility of residual drug effects. Age and education were not significantly different across the diagnostic groups.

\section{Results}

The mean (SD) cerebrospinal fluid HVA levels were: $40.9(17 \cdot 1) \mathrm{ng} / \mathrm{ml}$ for Alzheimer's disease, 34.6 (16) $\mathrm{ng} / \mathrm{ml}$ for Parkinson's disease, and $29.9(17.2) \mathrm{ng} / \mathrm{ml}$ for major depression. To define the pattern of cognitive deficits associated with dopamine deficiency, we compared patients with "low HVA" (9) to those with "high HVA" (12). Raw scores were residualised to correct for baseline differences in group means. ${ }^{9}$ (Group effect $=$ grand meangroup mean). The "low HVA" subset of patients, defined as those with HVA level below the mean of residualised scores included four with Alzheimer's disease, three with Parkinson's disease and two with major depressive disorder. The "low HVA" patients, regardless of clinical diagnosis, exhibited a distinctive pattern of performance on the neuropsychological tests selected to assess bradyphrenia, ability to benefit from structured tasks, and anomia (fig, table).

On the efficiency of processing speeded information (VIST), patients with low HVA performed significantly worse compared with high HVA patients $(t(16)=-2.31, \mathrm{p}<0.025)$ (one-tailed $t$-tests were used to test this directional hypothesis). On the Verbal Fluency Task, subjects with low HVA displayed significantly greater benefit from the structured condition compared with the unstructured condition $(t(16)=2 \cdot 86, p<0.01$, one-tailed). Patients in the low HVA group had slightly lower overall Mini Mental State Scores ( $t(16)$ $=-2.19, \mathrm{p}<0.025$, one-tailed). However, cognitive impairment in patients with low CSF HVA was not present in all domains. For example, scores on confrontation naming (Boston Naming Test) were not significantly impaired in patients with lower HVA compared with the high HVA group. Subjects in the low HVA group also had a significantly greater number of Parkinsonian motor features $(\mathrm{t}(16)=2.31, \mathrm{p}<0.025$, one-tailed) and more depression $(t(16)=2 \cdot 08, p<0 \cdot 05$, one-tailed $)$.

\section{Discussion}

In this study, patients with low CSF HVA, regardless of clinical diagnostic category, exhibited a characteristic profile of cognitive deficits, depressive symptoms, and extrapyramidal motor signs. Other investigators have described aspects of this neurobehavioural profile in subgroups of patients in each clinical category. ${ }^{810}$ This analysis draws together these aspects.

Research on cognitive deficits in Parkinson's disease provides the best comparison to the neuropsychological pattern described in the current study. Bachman and Albert ${ }^{1}$ emphasised the pattern of slowed thinking, impaired

Table Cognitive profiles of patients with low versus high CSF HVA

\begin{tabular}{|c|c|c|}
\hline & $\begin{array}{l}\text { High HVA } \\
(n=12)\end{array}$ & $\begin{array}{l}\text { Low } H V A \\
(n=9)\end{array}$ \\
\hline 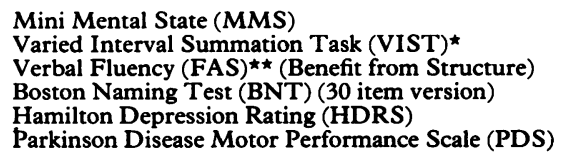 & $\begin{array}{l}23 \cdot 7,5 \cdot 45(79 \cdot 0,18 \cdot 2) \\
7 \cdot 8,3 \cdot 16(55 \cdot 7,22 \cdot 5) \\
0 \cdot 169,0 \cdot 234(16 \cdot 9,23 \cdot 4) \\
22 \cdot 4,5 \cdot 7(74 \cdot 7,19 \cdot 0) \\
8 \cdot 39,4 \cdot 9(11 \cdot 3,6 \cdot 6) \\
4 \cdot 76,3 \cdot 5(14 \cdot 87,10 \cdot 9)\end{array}$ & $\begin{array}{l}18 \cdot 9,4 \cdot 2 \dagger \dagger(63 \cdot 0,14 \cdot 0) \\
4 \cdot 89,2 \cdot 42 \dagger \dagger(34 \cdot 9,17 \cdot 3) \\
0 \cdot 494,0 \cdot 285 \dagger \dagger(49 \cdot 4,28 \cdot 5) \\
18 \cdot 6,6 \cdot 07(62 \cdot 0 \cdot 20 \cdot 2) \\
14 \cdot 1,7 \cdot 6 \dagger(19 \cdot 1,10 \cdot 3) \\
9 \cdot 82,6 \cdot 5(30 \cdot 7,20 \cdot 2)\end{array}$ \\
\hline
\end{tabular}


manipulation of acquired knowledge, and impaired memory, without aphasia. Studies measuring cognitive speed in Parkinson's disease and multiple sclerosis, and Huntington's disease often use the common term bradyphrenia, but methods to measure cognitive speed vary considerably. For example, cognitive speed has been measured using: speed of scanning elements in short term memory, ${ }^{11}{ }^{12}$ time to prepare a manual movement, ${ }^{11}$ difference in time to perform two digit symbol tasks which differed in cognitive complexity, ${ }^{10}$ attention and vigilance using a continuous performance task, ${ }^{8}$ reaction time on tasks differing in cognitive complexity ${ }^{13}$ and the Paced Auditory Serial Addition Test. ${ }^{14}$ In this pilot study, we used a simpler serial addition task (VIST) to disentangle motor speed and the efficiency of processing speeded information. Because the motor response from the patient was not timed, the score represents an individual's success at processing auditory stimuli at increasingly rapid intervals. However, this efficiency of processing speeded information cannot be equated with cognitive speed. Performance on this task is also dependent on motivation, auditory acuity, short term memory, distractibility, difficulty with calculation, and other factors.

Many investigators have reported decreased verbal fluency in Parkinson's disease ${ }^{1516}$ (using the FAS test), ${ }^{5}$ but have not specifically examined the benefit provided by semantic categories compared with phonemic categories. In this study, we observe a need for structure from semantic categories on a verbal fluency test. Comparable observations in the literature on Parkinson's disease include the description by Lees and Smith of "mental inflexibility", 15 and observations by Taylor et al ${ }^{17}$ of impairment in the ability to generate efficient strategies on spontaneously self-directed tasks.

Most reviews of dementia in Parkinson's disease agree that naming is relatively spared ${ }^{16}$ and not affected by levodopa treatment. ${ }^{18}$ Our findings that naming is relatively spared supports the hypothesis that only a specific set of cognitive functions is impaired and that we are not simply reporting a global cognitive decline.

The results of our study, as well as those from other laboratories, ${ }^{10} 19$ seem to support the earlier theory on the association between dopamine and cognitive impairment. ${ }^{1}$ Although there may be a role for mesolimbocortical dopamine neurons to account for this behaviour, other neurotransmitters have also been implicated, for example, in cognitive impairment in Parkinsonism. ${ }^{20}$ We have des- cribed a potential neurobehavioural "signature" associated with relatively low dopamine, but only the HVA level in CSF has been examined to date. It is likely that other neurotransmitter systems contribute to the neurobehavioural profile we have identified.

We thank Edith Kaplan for advice on the design of the neuropsychological evaluations, Errol Baker for statistical advice and Guila Glosser for comments on an earlier draft of this paper. This study was supported by NIH training fellowship paper. This study was supported by NIH training fellowship (NS07239-04), the Veterans Administration Medical Re
Service, and the Seidel Fund for the Study of Dementia.

1 Bachman DL, Albert ML. The dopaminergic syndromes of dementia. In: Pilleri G, Tagliavini F, eds. Cerebral aging and degenerative dementias. Brain Pathology, Vol 1. Waldau Bern, Switzerland: University of Berne, 1984:91-119.

2 McKhann G, Drachman D, Folstein M, Katzman R, Price D, Stadlan EM. Clinical diagnosis of Alzheimer's disease. Report of the NINCDS-ADRDA work group under the auspices of Department of Health and Human Services Task Force on Alzheimer's disease. Neurology (Cleveland) 1984;34:939-44.

3 American Psychiatric Association. Diagnostic and statistical manual for mental disorders, Third Ed, Washington, DC: APA, 1980

4 Gronwall DMA, Sampson H. The psychological effects of concussion. Auckland, NZ: Auckland University Press, 1974.

5 Benton AL, Hamsher KS. Multilingual aphasia examination. Iowa City: University of Iowa, 1976 (revised 1978)

6 Duvoisin RC. The evaluation of extrapyramidal disease. In Ajuriaguerra J, ed. Monoamines, noyaux gris centraux et syndrome de Parkinson. Paris: Masson, 1970:313-25.

7 Matson WR, Gamache PG, Beal MF, Bird ED. EC Array sensor concepts and data. Life Sci 1987;41:905-8.

8 Mayeux R, Stern Y, Sano M, Cote L, Williams JBW. Clinical and biochemical correlates of bradyphrenia in Parkinson's disease. Neurology 1987;37:1130-4.

9 Rosenthal R, Rosnow RL. Essentials of behavioral research. Methods and data analysis. New York: McGraw-Hill,

1984.
10 Rogers D, Lees AJ, Smith E, Trimble M, Stern GM. Bradyphrenia in Parkinson's disease and psychomotor retardation in depressive illness. An experimental study. Brain 1987;110:761-76.

11 Rafal RD, Posner MI, Walker JA, Friedrich FJ. Cognition and the basal ganglia: separating mental and motor components of performance in Parkinson's disease. Psychol Med 1984;16:257-65.

12 Wilson RS, Kaszniak AW, Klawans HL, Garron DC. High speed memory scanning in parkinsonism. Cortex 1980; 16:67-72.

13 Dubois B, Pillon B, Legault F, Agid Y, Lhermitte F Slowing of Cognitive Processing in Progressive Supranuclear Palsy. A Comparison with Parkinson's Disease. Arch Neurol 1988;45:1194-9.

14 Litvan I, Grafman J, Vendrell P, Martinez JM. Slowed Information Processing in Multiple Sclerosis. Arch Neurol 1988;45:281-5.

15 Lees AJ, Smith E. Cognitive deficits in the early stages of Parkinson's disease. Brain 1983;106:257-70.

16 El-Awar M, Becker JT, Hammond KM, Nebes RD, Boller F. Learning deficit in Parkinson's disease. Comparison with Alzheimer's disease and normal aging. Arch Neurol 1987;44:180-4.

17 Taylor AE, Saint-Cyr JA, Lang AE. Frontal lobe dysfunction in Parkinson's disease. Brain 1986;109:845-83.

18 Delis D, Direnfeld L, Alexander MP, Kaplan E. Cognitive fluctuations associated with on-off phenomenon in Parkinson's disease. Neurology 1982;32:1049-52.

19 Kaye JA, Kay AD, May C, et al. Cerebrospinal fluid monoamine markers are reduced in dementia of the monoamine markers are reduced in dementia of the Alzheimer's type with

20 Pillon B, Dubois B, Cusimano G, Bonnet AM, Lheymitte F, Agid Y. Does cognitive impairment in Parkinson's disease result from non-dopaminergic lesions? J Neurol Neurosurg Psychiatry 1989;52:201-6. 